INPLASY

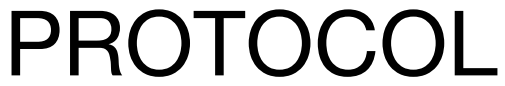

To cite: Peng et al. Tuina for diabetes with obesity: Protocol for a systematic review and meta-analysis. Inplasy protocol 2020110106. doi:

10.37766/inplasy2020.11.0106

Received: 24 November 2020

Published: 24 November 2020

Corresponding author:

Sihan Peng

476674985@qq.com

Author Affiliation:

Hospital of Chengdu University of Traditional Chinese

Medicine

Support: 19ZL02.

Review Stage at time of this submission: The review has not yet started.

Conflicts of interest:

The authors declare no conflict of interest.

\section{Tuina for diabetes with obesity: Protocol for a systematic review and} meta-analysis

Peng, S1; Xie, Z2; Zhang, X3; Liu, Y4; Zhang, X5; Liang, X6; Wang, $\mathrm{H}^{7} ; \mathrm{Xie}, \mathrm{C}^{8}$.

Review question / Objective: P:diabetes with obesity; I:Tuina therapy; C: other forms of Chinese traditional non-drug intervention such as acupuncture, Qigong, etc; O: the main outcomes of this review are fasting blood glucose (FBG), two hour postprandian blood glucose (2hPG) and the weight. The additional outcomes include $\mathrm{HbA1c}$, reduced symptom scores, etc; S: randomized controlled clinical trials and quasirandomized controlled trials.

Condition being studied: All researchers have rich experience in literature review and data analysis.

Information sources: We will search the following electronic databases: Pubmed, Embase, Cochrane Library, Web of science, Chinese National Knowledge Infrastructure (CNKI), Sino Med, Wanfang, Chinese Clinical Trial Registry System, China Biomedical Literature Database (CBM).The time limit for retrieving studies is from establishment to November 2020 for each database. Randomized controlled clinical trials related to Tuina intervention on diabetes with obesity will be included. Data synthesis, sensitivity analysis, subgroup analysis as well as the assessment of bias risk will be conducted by using Stata V.13.0 and Review manager 5.3 software.

INPLASY registration number: This protocol was registered with the International Platform of Registered Systematic Review and Meta-Analysis Protocols (INPLASY) on 24 November 2020 and was last updated on 24 November 2020 (registration number INPLASY2020110106).

\section{INTRODUCTION}

Review question / Objective: P:diabetes with obesity; I:Tuina therapy; C: other forms of Chinese traditional non-drug intervention such as acupuncture, Qigong, etc; O: the main outcomes of this review are fasting blood glucose (FBG), two hour postprandian blood glucose (2hPG) and the weight. The additional outcomes include 
HbA1c, reduced symptom scores, etc; S: randomized controlled clinical trials and quasi-randomized controlled trials.

Condition being studied: All researchers have rich experience in literature review and data analysis.

\section{METHODS}

Search strategy: We will search the studies published on Pubmed, Embase, Cochrane Library, Web of science, Chinese National Knowledge Infrastructure (CNKI), Sino Med, Wanfang, Chinese Clinical Trial Registry System, China Biomedical Literature Database (CBM), Since the inception to November 2020 without language restrictions.

Participant or population: Patients with diabetes and obesity.

Intervention: This meta-analysis will include the RCTs of Tuina therapy regardless of duration and frequency.

Comparator: Other forms of Chinese traditional non-drug intervention such as acupuncture, Qigong, etc; Conventional treatment according to relevant guideline, or other forms of Chinese traditional nondrug intervention such as Tai Chi, acupuncture, Tuina, etc.

Study designs to be included: Randomized controlled clinical trials and quasirandomized controlled trials will be considered for inclusion in this study. Studies involving non-RCTs, reviews, animal experiments, case series will be excluded.

Eligibility criteria: Patients diagnosed with diabetes and obesity(aged $\geq 18$ years)will be included in this study. There is no limitation on the gender, ethnicity and nation.

Information sources: We will search the following electronic databases: Pubmed, Embase, Cochrane Library, Web of science, Chinese National Knowledge Infrastructure (CNKI), Sino Med, Wanfang, Chinese Clinical Trial Registry System, China
Biomedical Literature Database (CBM).The time limit for retrieving studies is from establishment to November 2020 for each database. Randomized controlled clinical trials related to Tuina intervention on diabetes with obesity will be included. Data synthesis, sensitivity analysis, subgroup analysis as well as the assessment of bias risk will be conducted by using Stata V.13.0 and Review manager 5.3 software.

Main outcome(s): The main outcomes of this review are fasting blood glucose (FBG), two hour postprandian blood glucose (2hPG) and the weight.

Additional outcome(s): The additional outcomes include HbA1c, reduced symptom scores, etc.

Quality assessment / Risk of bias analysis: The risk of bias will be assessed independently according to the Cochrane Handbook for Systematic Reviews of Interventions by 2 reviewers. Random sequence generation, allocation concealment, blinding of participants and personnel, the blindness of outcome assessments, incomplete outcome data, selective outcome reporting, and other bias will be evaluated as low risk, high risk, or ambiguous risk in each RCT. The results will be checked repeatedly and the disagreement will be resolved by further discussion of all investigators.

Strategy of data synthesis: The Review Manage software will be utilized to analyze all data. We will calculate the risk ratio (RR) for dichotomous with $95 \%$ confidence intervals (Cls), And the mean difference (MD) will be included in the meta-analysis for continuous data. While the outcome variables are measured by different scales, standard mean differences (SMD) analysis with $95 \% \mathrm{Cl}$ will also be estimated in the meta-analysis.

Subgroup analysis: Subgroup analysis will be performed to evaluate the high heterogeneity of included researches. We will conduct subgroup analysis based on the different acupuncture points for Tuina, duration of Tuina, frequency of Tuina, etc. 
Sensibility analysis: Sensitivity analysis will be required if there are possible low-quality studies after the quality assessment of the included researches. We will observe fluctuation of termination by changing the type of research and reanalysis of the indistinct data.

Country(ies) involved: China.

Keywords: Tuina, meta-analysis, protocol, diabetes with obesity, systematic review.

Contributions of each author:

Author 1 - Sihan Peng.

Author 2 - Ziyan Xie.

Author 3 - Xiyu Zhang.

Author 4 - Ya Liu.

Author 5 - Xiangeng Zhang.

Author 6 - Xiaoli Liang.

Author 7 - Hongyan Wang.

Author 8 - Chunguang Xie. 